\title{
Clinical characteristics and survival of Chinese patients diagnosed with pulmonary arterial hypertension who carry BMPR2 or EIF2KAK4 variants
}

Qixian Zeng ${ }^{1 \dagger}$, Hang Yang ${ }^{2 \dagger}$, Bingyang Liu ${ }^{1 \dagger}$, Yanyun $\mathrm{Ma}^{2}$, Zhihong Liu', Qianlong Chen², Wenke Li , Qin Luo', Zhihui Zhao', Zhou Zhou ${ }^{2^{*}}$ and Changming Xiong ${ }^{1 *}$

\begin{abstract}
Background: Variants in the gene encoding bone morphogenetic protein receptor type II (BMPR2) are the most common genetic cause of pulmonary arterial hypertension (PAH), whereas biallelic variants in the eukaryotic translation initiation factor 2 alpha kinase 4 gene (EIF2AK4) are described in pulmonary veno-occlusive disease/ pulmonary capillary haemangiomatosis (PVOD/PCH). Racial background may influence the clinical characteristics of patients diagnosed with $\mathrm{PAH}$ or PVOD/PCH. Here, we compared the clinical characteristics and survival between patients with BMPR2 variants or EIF2AK4 variants in a Chinese population.

Methods: Heterozygous variants in BMPR2 and homozygous or compound heterozygous biallelic EIF2AK4 variants predicted to be deleterious were identified as potentially causal. Clinical and radiological data were collected and analysed. The primary outcomes were death or lung transplantation. Hazard ratios (HRs) for death or transplantation associated with the presence of BMPR2 or biallelic EIF2AK4 variants were calculated using Cox proportional hazards models to analyse patient survival.

\footnotetext{
* Correspondence: zhouzhou@fuwaihospital.org; xiongcmfw@163.com

${ }^{\dagger}$ Qixian Zeng, Hang Yang and Bingyang Liu contributed equally to this work.

${ }^{2}$ State Key Laboratory of Cardiovascular Disease, Beijing Key Laboratory for

Molecular Diagnostics of Cardiovascular Disease, Diagnostic Laboratory

Service, Fuwai Hosptial, National Center for Cardiovascular Diseases, Chinese

Academy of Medical Sciences and Peking Union Medical College, North Lishi

Road, Xicheng District No.167, Beijing, China

${ }^{1}$ State Key Laboratory of Cardiovascular Disease, Center of Pulmonary

Vascular Disease, Fuwai Hospital, National Center for Cardiovascular Disease, Chinese Academy of Medical Sciences and Peking Union Medical College,

North Lishi Road, Xicheng District, No.167, Beijing, China
}

C C The Author(s). 2020 Open Access This article is licensed under a Creative Commons Attribution 4.0 International License, which permits use, sharing, adaptation, distribution and reproduction in any medium or format, as long as you give appropriate credit to the original author(s) and the source, provide a link to the Creative Commons licence, and indicate if changes were made. The images or other third party material in this article are included in the article's Creative Commons licence, unless indicated otherwise in a credit line to the material. If material is not included in the article's Creative Commons licence and your intended use is not permitted by statutory regulation or exceeds the permitted use, you will need to obtain permission directly from the copyright holder. To view a copy of this licence, visit http://creativecommons.org/licenses/by/4.0/ The Creative Commons Public Domain Dedication waiver (http://creativecommons.org/publicdomain/zero/1.0/) applies to the data made available in this article, unless otherwise stated in a credit line to the data. 


\begin{abstract}
(Continued from previous page)
Results: Two hundred thirty-two patients with PAH were enrolled for genetic testing, and PAH patients with associated conditions were excluded from the study. Forty-five patients with BMPR2 variants and 11 patients with biallelic EIF2AK4 variants were recruited. PAH patients with BMPR2 or biallelic EIF2AK4 variants presented symptoms at the ages of $25.57 \pm 10.17$ years and $31.6 \pm 9.38$ years, respectively. The whole group of patients showed female dominance either with BMPR2 variants or biallelic EIF2AK4 variants. Specific radiological abnormalities are more prominent in EIF2AK4 variant carriers but can also be found in some patients with BMPR2 variants. Biallelic EIF2AK4 variant carriers had worse survival than BMPR2 variant carriers $(p<0.0001)$.

Conclusions: Clinical pictures of PAH patients with BMPR2 and biallelic EIF2AK4 variants in the Chinese population differ from other populations by a younger age at diagnosis and demonstrate female dominance in the whole patient group. High-resolution chest CT can help assist in differentiating PAH with PVOD/PCH. BMPR2 variants and biallelic EIF2AK4 variants are associated with adverse outcomes, but the survival of patients with biallelic EIF2AK4 variants is dismal.
\end{abstract}

Keywords: BMPR2 variants, Biallelic EIF2AK4 variants, PVOD/PCH, Survival

\section{Background}

Pulmonary arterial hypertension (PAH) is a devastating disorder ultimately leading to increased pulmonary vascular resistance and right ventricular failure [1]. Variants in the gene encoding the bone morphogenetic protein type 2 receptor $(B M P R 2)$ are the most common genetic cause of PAH. Approximately $14.5-20 \%$ of idiopathic (IPAH) patients and $53.3-80 \%$ of heritable $(\mathrm{HPAH}) \mathrm{pa}-$ tients harbour variants at the BMPR2 locus from different populations [2-5]. Patients with $\mathrm{PAH}$ and BMPR2 variants present at a younger age with more severe disease and are at increased risk of death compared with those without $B M P R 2$ variants [3]. However, variants in $B M P R 2$ have also been reported in patients with histologically proven pulmonary veno-occlusive disease (PVOD) and pulmonary capillary haemangiomatosis $(\mathrm{PCH})[6,7]$, which are rare forms of $\mathrm{PAH}$ under the current classification [1].

A significant advance in the molecular diagnosis of PVOD/PCH was the finding of biallelic variants in the gene encoding eukaryotic translation initiation factor 2 alpha kinase 4 (EIF2AK4) [8, 9]. Pathogenic biallelic EIF2AK4 variants are rarely identified $(<2 \%)$ in patients diagnosed clinically with IPAH $[10,11]$. It is suggested that EIF2AK4 variants are specific to $\mathrm{PVOD} / \mathrm{PCH}$ and that finding biallelic EIF2AK4 variants in a patient with pulmonary hypertension would be diagnostic of PVOD/ $\mathrm{PCH}$. Patients with $\mathrm{PVOD} / \mathrm{PCH}$ have a poor prognosis and risk fatal pulmonary oedema with the use of pulmonary artery vasodilator therapies [7, 12]. Given that both BMPR2 variant carriers and biallelic EIF2AK4 variant carriers have poor prognoses, the different clinical phenotypes of the above gene variant carriers have not been fully clarified in all populations [11].

Previous investigators have extensively described the clinical characteristics of patients diagnosed with $\mathrm{PAH}$ who carry $B M P R 2$ variants or $\mathrm{PVOD} / \mathrm{PCH}$ who carry biallelic EIF2AK4 variants in European and American populations, but Chinese populations are not well represented in these reports [12, 13]. Ethnic background may influence the clinical characteristics of patients diagnosed with PAH or PVOD/PCH $[2,14]$. Here, we report the clinical characteristics of patients diagnosed with PAH who carry BMPR2 or biallelic EIF2AK4 variants in a Chinese PAH population, and long-term survival was also compared.

\section{Methods}

\section{Ethical approval and consent}

The study was approved by the ethics committees of Fuwai Hospital (Approval No: 2017-877) and adhered to the Declaration of Helsinki. All patients recruited to the present study provided written informed consent for genetic analysis and the capture of clinical data.

\section{Recruitment and patients}

The diagnosis of PAH was made by using pulmonary artery catheterization to confirm a mean pulmonary arterial pressure $(\mathrm{mPAP}) \geq 25 \mathrm{mmHg}$, pulmonary capillary wedge pressure (PCWP) $\leq 15 \mathrm{mmHg}$, and pulmonary vascular resistance $(\mathrm{PVR})>3$ Wood units. Additional clinical information (e.g., history of exposure to anorexigens) was collected, and diagnostic testing excluded associated PAH and alternative diagnoses (e.g., chronic thromboembolic pulmonary hypertension). Patients with idiopathic $\mathrm{PAH}$, familial $\mathrm{PAH}$ and $\mathrm{PVOD} / \mathrm{PCH}$ who were clinically diagnosed according to international guidelines by a specialist at Fuwai Hospital pulmonary vascular centre in China between November 2015 and June 2019 were recruited. Throughout this article, we classify patients as having idiopathic PAH or familial $\mathrm{PAH}$ on the basis of the absence or presence of a family history of the disease, respectively. 


\section{Genetic analysis}

The detailed genetic analysis method was previously described, and we briefly describe it here [4]. A customdesigned gene panel with 13 well-associated PAH genes was used for gene testing. This gene panel was $87 \mathrm{~KB}$ with $98.7 \%$ coverage of the target regions. These 13 genes include $B M P R 2, K C N K 3$ (potassium two-pore domain channel subfamily $\mathrm{K}$ member 3 ), $C A V 1$ (caveolin 1), SMAD9 (SMAD family member 9), BMPR1B (bone morphogenetic protein receptor type 1B), ACVRL1 (activin A receptor-like type 1), ENG (endoglin), GDF2 (growth differentiation factor 2), SMAD4 (SMAD family member 4), EIF2AK4, KCNA5 (potassium voltage-gated channel subfamily A member 5), NOTCH3 (notch 3) and TOPBP1 (DNA topoisomerase II binding protein 1). Variants with a minor allele frequency of $>0.1 \%$ in panel genes other than EIF $2 A K 4$ were excluded as polymorphism variants, whereas $E I F 2 A K 4$ variants with a minor allele frequency of $>1 \%$ were excluded due to an autosomal-recessive inherited pattern. The remaining variants were classified into five categories according to the recommendations of the American College of Medical Genetics: benign, likely benign, unknown significance, likely pathogenic and pathogenic. Only likely pathogenic and pathogenic variants of BMPR2 or $E I F 2 A K 4$ variants were included for further analysis.

\section{Clinical data collection and follow-up}

Patients' clinical records were reviewed to capture phenotypic variables from the time of diagnosis and followed-up for a median period of 22.77 months. Haemodynamic evaluation by right heart catheterization was performed at baseline in all patients. PCWP, mPAP, mixed venous oxygen saturation (SvO2) and arterial oxygen saturation $(\mathrm{SaO} 2)$ were collected. Cardiac output (CO) was measured using the standard Fick technique. Baseline haemodynamic data and response to short-term vasodilator iloprost $(20 \mu \mathrm{g}$ for $10 \mathrm{~min})$ were obtained. Vasodilator responsiveness was defined as a reduction in mPAP of at least $10 \mathrm{mmHg}$ compared with baseline and attaining a level less than $40 \mathrm{mmHg}$ with no reduction in $\mathrm{CO}$ after administration of inhaled iloprost.

Patients received follow-up every 6 months through telephone or clinic visits. The primary outcome was the composite of death or lung transplantation. Outcomes were censored if a patient was lost to follow-up or reached the end of the follow-up period. The date of $\mathrm{PAH}$ diagnosis was defined as the date of diagnostic right heart catheterization.

\section{Statistical analysis}

Baseline characteristics of patients according to $B M P R 2$ or EIF2AK4 variants were compared using t tests for continuous variables and $\chi^{2}$ tests for categorical variables. Associations of $B M P R 2$ or biallelic EIF $2 A K 4$ variant status with risk of death or transplantation recorded during follow-up were assessed using Cox proportional hazards regression models. Survival curves comparing patients with BMPR2 variants or biallelic EIF2AK4 variants were calculated using unadjusted Kaplan-Meier estimates and compared using the logrank test (GraphPad Software, Inc). A two-sided $p$ value less than 0.05 was considered statistically significant throughout the study. Statistical analyses were performed using SPSS (version 23; IBM Corp, 2015).

\section{Results}

\section{Study patients}

In total, genetic analysis was performed on $232 \mathrm{PAH}$ patients in the study. Fifteen patients diagnosed with associated PAH were excluded, and a total of 217 patients underwent further analysis. Of 217 PAH patients, 22 patients from 19 families exhibited confirmed familial aggregation of the disease, and 195 patients with negative family histories were classified as idiopathic PAH cases.

Predicted deleterious BMPR2 variants were found in 12 probands (63\%) with familial PAH and 41 patients (21\%) with idiopathic PAH. Seven (including two siblings from one family) of the 19 patients (36.8\%) with clinical diagnosis of PVOD/PCH carried biallelic EIF2AK4 variants (1 homozygote and 6 compound heterozygotes). Six EIF2AK4 variants were also found in 4 patients diagnosed clinically with $\mathrm{PAH}$, in whom there was no clinical suspicion of PVOD/ $\mathrm{PCH}$ ( 1 homozygote and 3 heterozygotes). Of the 11 biallelic EIF2AK4 variant carriers, 3 were familial cases (2 families), 8 were sporadic cases, and no history of solvent or chemotherapy exposure was noted. Notably, no gene defects were detected in 4 patients with $\mathrm{HPAH}$ in our cohort, and further genetic testing for the identification of new genes potentially related to $\mathrm{PAH}$ is in progress.

Therefore, 45 patients carrying likely pathogenic or pathogenic $B M P R 2$ variants and 11 patients carrying biallelic EIF2AK4 variants (see Supplement Table 1 \& 2, some data have been reported.) with intact clinical data underwent further examination in the study.

\section{Clinical features of PAH carriers with $B M P R 2$ or biallelic EIF2AK4 variants}

Patients with a clinical diagnosis of $\mathrm{PAH}$ and with $B M P R 2$ or biallelic EIF2AK4 variants presented with symptoms at the age of $25.57 \pm 10.17$ years and $31.6 \pm$ 9.38 years, respectively. The whole group of patients showed female dominance with BMPR2 variants and with biallelic EIF2AK4 variants (Table 1).

All patients had advanced NYHA functional class at presentation, as well as severe functional impairments. However, no significant differences in $\mathrm{N}$-terminal pro-Btype natriuretic peptide (NT-proBNP) value, 6-min walk 
Table 1 Demographics and clinical characteristics of patients at diagnosis with BMPR2 and with biallelic EIF2AK4 variants

\begin{tabular}{|c|c|c|c|}
\hline & $B M P R 2(N=45)$ & EIF2AK4 $(N=11)$ & $p$ value \\
\hline F/M, (F\%) & $30 / 15(66.7 \%)$ & $8 / 3(72.7 \%)$ & 0.7 \\
\hline Age at diagnosis, y & $25.57 \pm 10.17$ & $31.6 \pm 9.38$ & 0.09 \\
\hline $\mathrm{BMI}, \mathrm{kg} / \mathrm{m}^{2}$ & $21.77 \pm 3.99$ & $20.78 \pm 3.49$ & 0.49 \\
\hline \multicolumn{4}{|l|}{ NYHA functional class } \\
\hline$|-| \mid(n / \%)$ & $21 / 46.7 \%$ & $5 / 45.5 \%$ & 0.94 \\
\hline$\|||(n, \%)$ & $22 / 48.9 \%$ & $5 / 45.5 \%$ & 0.84 \\
\hline IV(n, \%) & $2 / 4.4 \%$ & $1 / 9.1 \%$ & 0.54 \\
\hline NT-proBNP, pg/ml & $1806.41 \pm 1466.61$ & $1703.5 \pm 1778.76$ & 0.85 \\
\hline DLCO, predicted \% & $67.74 \pm 14.63$ & $29.88 \pm 6.01$ & $0.00^{*}$ \\
\hline Peak VO2, predicted \% & $32.56 \pm 12.83$ & $29.12 \pm 9.70$ & 0.48 \\
\hline $6 \mathrm{MWD}, \mathrm{m}$ & $365.77 \pm 96.11$ & $390.56 \pm 130.86$ & 0.53 \\
\hline $\mathrm{SvO} 2, \%$ & $64.19 \pm 6.96$ & $70.87 \pm 7.34$ & $0.02^{*}$ \\
\hline $\mathrm{SaO} 2, \%$ & $95.11 \pm 3.54$ & $92.00 \pm 2.84$ & $0.03^{*}$ \\
\hline $\mathrm{RAP}, \mathrm{mmHg}$ & $5.11 \pm 3.99$ & $4.29 \pm 2.69$ & 0.61 \\
\hline mPAP, mmHg & $63.62 \pm 14.97$ & $51.00 \pm 4.24$ & 0.00 \\
\hline PAWP, mmHg & $7.15 \pm 3.45$ & $7.57 \pm 2.99$ & 0.77 \\
\hline PVR, Wood units & $18.85 \pm 6.77$ & $12.03 \pm 2.00$ & $0.01^{*}$ \\
\hline $\mathrm{Cl}, \mathrm{L} / \mathrm{min} / \mathrm{m} 2$ & $2.39 \pm 0.52$ & $3.24 \pm 1.04$ & 0.07 \\
\hline Vasodilator responder & 0 & $1 / 9 \%$ & - \\
\hline
\end{tabular}

Values are shown as mean \pm SD or $\mathrm{n}(\%)$. BMI body mass index, NT-proBNP Nterminal pro-B-type natriuretic peptide, DLCO diffusing lung capacity of carbon monoxide, PeakVO2 peak oxygen consumption, 6MWD 6 min walk distance, $\mathrm{SvO} 2$ mixed venous oxygen saturation, $\mathrm{SaO} 2$ arterial oxygen saturation, RAP right atrial pressure, $M P A P$ mean pulmonary artery pressure, $P A W P$ pulmonary artery wedge pressure, $P V R$ pulmonary vascular resistance, $C l$ cardiac index *:p $<\mathbf{0 . 0 5}$

distance (6MWD) and peak oxygen consumption when expressed as percentage predicted (PeakVO2\%) adjusted for age, height, and sex were found between the two groups. Notably, patients with biallelic EIF2AK4 variants exhibited a reduced diffusing lung capacity of carbon monoxide (DLCO, [29.88 \pm 6.01$] \%$ predicted) compared with $B M P R 2$ variant carriers $([67.74 \pm 14.63] \%$ predicted; $p=0.00)$.

Right heart catheterization at diagnosis showed severe pre-capillary pulmonary hypertension in both groups. Haemodynamic variables, including $\mathrm{SvO} 2$, PVR and cardiac index $(\mathrm{CI})$, were more severely impaired in carriers with $B M P R 2$ variants than in carriers with biallelic EIF2AK4 variants (Table 1). Acute vasodilator testing was performed in both groups without acute pulmonary oedema complications. One (9\%) of 11 carriers of biallelic EIF2AK4 variant carriers fulfilled the haemodynamic criteria for significant acute vasoreactivity, while none of the $B M P R 2$ carriers responded to acute vasodilators (Table 1).

\section{CT features of PAH carriers with BMPR2 or biallelic EIF2AK4 variants}

High-resolution Computed Tomography (CT) of the chest was available in 38 BMPR2 variant carriers and 11 biallelic EIF2AK4 variant carriers. Centrilobular ground glass opacification, interlobular septal thickening and mediastinal lymphadenopathy are considered suggestive signs of PVOD/PCH. The characteristic CT signs were more prominent in biallelic EIF2AK4 variant carriers than in BMPR2 variant carriers. Specifically, we found centrilobular ground glass opacification in 10 (90.9\%), interlobular septal thickening in $9(81.8 \%)$, and mediastinal lymphadenopathy in $5(45.5 \%)$ patients carrying biallelic EIF2AK4 variants (Table 2). More than $80 \%$ of biallelic EIF2AK4 variant carriers presented with at least two of these radiological abnormalities at diagnosis, and $4(36.4 \%)$ displayed all three radiological abnormalities. In contrast, such characteristic CT features were less commonly presented in patients carrying BMPR2 variants. Twenty-four (63.2\%) PAH patients with BMPR2 variants demonstrated no radiological abnormalities on high-resolution CT. However, three (7.9\%) BMPR2 variant carriers presented with all three radiological abnormalities, whose percentage was significantly lower compared with $36.4 \%$ of patients with biallelic EIF2AK4 variants, $p=0.0017$.

\section{Survival of patients with BMPR2 or biallelic EIF2AK4 variants}

Most of the patients with $B M P R 2$ variants received mono- or combined specific medical therapy for PAH (see Table 3), while $18.2 \%$ of patients with EIF2AK4 variant carriers received no $\mathrm{PAH}$-specific therapy.

Patients diagnosed clinically as having PAH with biallelic EIF2AK4 variants exhibited a shorter survival time from diagnosis compared with the BMPR2 variant carriers $(p<0.0001$ for log-rank test, Fig. 1). BMPR2 or biallelic EIF2AK4 variants, low DLCO, low Peak VO2\% and reduced 6MWD were associated with an increased risk for death or lung transplant (Table 4). However, no significant effects of haemodynamic measurements on survival were observed in the present study.

\section{Discussion}

Substantial progress has been made in understanding the clinical characteristics based on various PAH genetic backgrounds during the past decade from different populations $[2-4,10-12,14-16]$. The present study provides data for a Chinese population describing the clinical and radiological features of PAH patients carrying either pathogenic $B M P R 2$ variants or pathogenic biallelic EIF2AK4 variants.

Previous studies have reported that $B M P R 2$ variants are the most common genetic cause of PAH $[1,3,15]$. As expected, pathogenic BMPR2 variants were most commonly found in patients with a clinical diagnosis of HPAH or idiopathic PAH in the present study. In addition, we identified a high frequency of biallelic 
Table 2 Radiological Features of Patients with BMPR2 mutations or biallelic EIF2AK4 variants

\begin{tabular}{|c|c|c|c|}
\hline & $\begin{array}{l}\text { BMPR2 }(N=38) \\
\mathrm{n}(\%)\end{array}$ & $\begin{array}{l}\text { EIF2AK4 }(N=11) \\
\mathrm{n}(\%)\end{array}$ & $p$ value \\
\hline Centrilobular ground glass opacification & $13(34.2)$ & 10(90.9) & $0.001 *$ \\
\hline Interlobular septal thickening & $11(28.9)$ & $9(81.8)$ & $0.002^{*}$ \\
\hline Mediastinal lymphadenopathy & $4(10.5)$ & $5(45.5)$ & $0.008^{*}$ \\
\hline Three signs & $3(7.9)$ & $4(36.4)$ & $0.017^{*}$ \\
\hline Two signs & $8(21.1)$ & $5(45.5)$ & 0.11 \\
\hline One sign & $3(7.9)$ & $2(18.2)$ & 0.32 \\
\hline None & $24(63.2)$ & 0 & - \\
\hline
\end{tabular}

Abbreviations see Table 1

EIF2AK4 variants in patients with a clinical presentation of $\mathrm{PVOD} / \mathrm{PCH}$. However, we also found biallelic EIF2AK4 variants in patients with a clinical diagnosis of $\mathrm{PAH}$, which was consistent with other observations [11]. Therefore, gene testing for $B M P R 2$ variant detection is important in all eligible PAH patients. However, for a $\mathrm{PAH}$ patient with clinical suspicion of PVOD/PCH, biallelic EIF2AK4 variant testing is recommended, regardless of familial history. The discovery of biallelic EIF2AK4 variants in $\mathrm{PVOD} / \mathrm{PCH}$ raised the possibility of rapid molecular diagnosis in patients with familial and sporadic PVOD/PCH. In the present study, the presence of biallelic EIF2AK4 variants was associated with a poor prognosis compared to $\mathrm{PAH}$ patients with $B M P R 2$ variants. Therefore, genetic testing can help identify patients with pathogenic EIF2AK4 variants early and may facilitate timely PVOD/PCH diagnosis and referral for lung transplantation before their conditions deteriorate.

The presence of BMPR2 variants or biallelic EIF2AK4 variants possesses similar demographic characteristics and clinical presentations in the present study. As we noted, all patients presented with clinical, functional and haemodynamic impairments regardless of whether they were BMPR2 variant carriers or EIF2AK4 variant carriers. However, in contrast to previous studies [11, 12], patients with BMPR2 or biallelic EIF2AK4 variants were at a younger age when diagnosed in our study, although clinical and haemodynamic inclusion criteria were similar. It is interesting to note that in a previous study from China, the authors also found the mean age of patients was younger in a Chinese Han cohort than the age of patients with IPAH or HPAH in other populations [2]. Although it is not easy to speculate the reason for such a difference, it may possibly be ascribed to an increasing pressure of geographic- and population-specific factors.

Another striking result is that the predominant proportion of females affected by EIF2AK4 variants contrasts with results reported by Montani et al., who showed an equal number of men and women affected by EIF2AK4 variants from different populations [12]. We acknowledge that it is difficult to speculate the reason for the diversity based on such a small population. However, possible explanations include increased representation of ethnic groups with a higher female: male ratio in Chinese PAH patients with biallelic EIF2AK4 variants. This may also be ascribed to other race-specific factors and environmental backgrounds. However, further largescale investigation is needed to verify these observations.

Moreover, it is worth noting that patients with biallelic EIF2AK4 variants demonstrated a significantly reduced DLCO compared with patients with BMPR2 variants. The distinctive CT features with centrilobular ground glass opacification and interlobular septal thickening indicate thickening of the blood-gas barrier, which may lead to widespread reduction in alveolar gas exchange

Table 3 Specific Medical therapy for PAH with BMPR2 mutations or biallelic EIF2AK4 variants

\begin{tabular}{lll}
\hline & $B M P R 2(N=45)$ & EIF2AK4 (N=11) \\
\hline ERA monotherapy & $5(11.1)$ & 0 \\
PDE5 inhibitor monotherapy & $21(46.7)$ & $5(45.5)$ \\
Prostacyclin derivative monotherapy & $1(2.2)$ & $1(9.1)$ \\
ERA plus PDE5 & $14(31.3)$ & $3(27.3)$ \\
ERA plus prostacyclin derivative & $1(2.2)$ & 0 \\
PDE5 inhibitor plus prostacyclin derivative & $1(2.2)$ & 0 \\
ERA plus PDE5 inhibitor plus prostacyclin derivative & $1(2.2)$ & 0 \\
No specific PAH therapy & $1(2.2)$ & $2(18.2)$ \\
\hline
\end{tabular}

ERA endothelin receptor antagonist, PDE5 phosphoriesterase type 5 


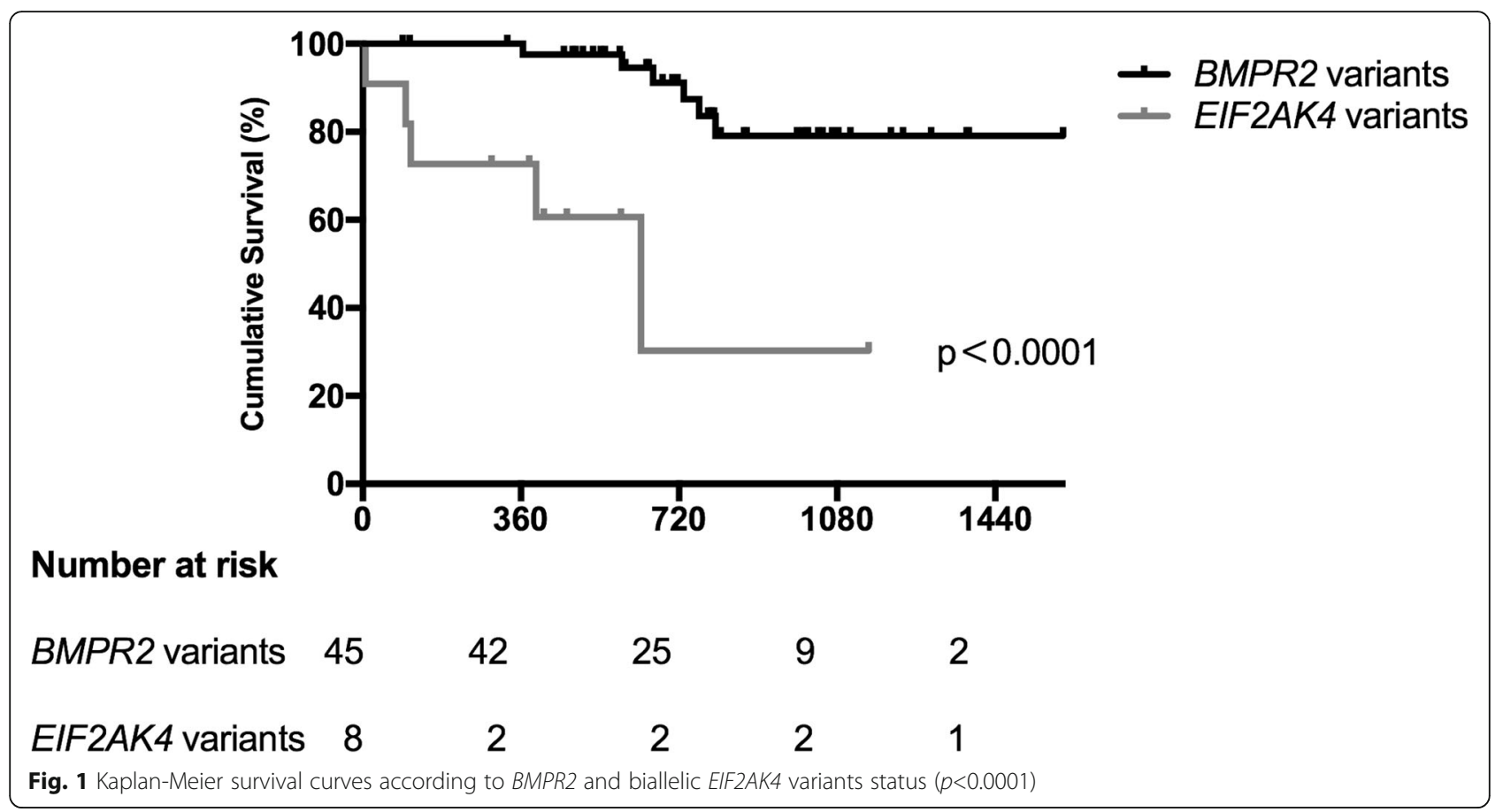

and thus reduction in DLCO in biallelic EIF2AK4 variant carriers.

It is generally recognized that high-resolution CT imaging is a useful noninvasive method to assist in the diagnosis of suspected PVOD/PCH and direct the patient towards specialist review [17]. In keeping with previous studies, we found an increased prevalence of centrilobular ground glass opacification, interlobular septal thickening and mediastinal lymphadenopathy in patients with biallelic EIF2AK4 variants. We also noted that approximately one-third of biallelic EIF2AK4 variant carriers demonstrated all three distinctive CT features, indicating differing radiological features of similar genetic backgrounds in various individuals. In addition, we found some patients with $B M P R 2$ variants revealing diffuse ground glass opacity with thickening interlobular

Table 4 Proportion of excess risk mediated by genetic status and clinical variables at diagnosis

\begin{tabular}{lll}
\hline Death or transplantation & HR $(95 \%$ Cl) & $p$ value \\
\hline BMPR2 or biallelic EIF2AK4 variants & $0.114(0.032-0.404)$ & $0.001^{*}$ \\
Adjusted for DLCO & $0.035(0.001-1.032)$ & 0.05 \\
Adjusted for DLCO, Peak VO2\%, 6MWD & $0.001(0.000-0.179)$ & $0.011^{*}$ \\
DLCO & $0.958(0.919-0.999)$ & $0.042^{*}$ \\
Adjusted for BMPR2 or biallelic EIF2AK4 variants & $1.024(0.954-1.09)$ & 0.509 \\
Adjusted for Peak VO2\%, 6MWD, BMPR2 or EIF2AK4 mutations & $1.158(1.002-1.339)$ & $0.047^{*}$ \\
Peak VO2\% & $0.89(0.819-0.987)$ & $0.025^{*}$ \\
Adjusted for BMPR2 or biallelic EIF2AK4 variants & $0.883(0.803-0.971)$ & $0.011^{*}$ \\
Adjusted for DLCO, 6MWD, BMPR2 or EIF2AK4 mutations & $0.725(0.505-1.039)$ & 0.08 \\
6MWD & $0.992(0.984-1)$ & $0.04^{*}$ \\
Adjusted for BMPR2 or biallelic EIF2AK4 variants & $0.992(0.985-0.999)$ & $0.018^{*}$ \\
Adjusted for DLCO, Peak VO2\%, BMPR2 or biallelic EIF2AK4 variants & $1.00(0.988-1.013)$ & 0.975 \\
Cardiac Index & $0.778(0.285-2.122)$ & 0.624 \\
Pulmonary vascular resistance & $1(0.999-1.001)$ & 0.429 \\
Mean pulmonary arterial pressure & $1.013(0.971-1.057)$ & 0.55 \\
SvO2 & $1.009(0.923-1.1037)$ & 0.841 \\
\hline
\end{tabular}

HR Hazard ratios, other abbreviations see Table 1 
septa in high-resolution $\mathrm{CT}$, which might be misdiagnosed as $\mathrm{PVOD} / \mathrm{PCH}$. This was similar to a previous study, in which a radiological suspicion of $\mathrm{PVOD} / \mathrm{PCH}$ was presented in $71 \%$ of those with $\mathrm{PVOD} / \mathrm{PCH}, 57 \%$ of patients with a clinical diagnosis of PAH and biallelic EIF2AK4 variants, $14 \%$ of patients with $\mathrm{PAH}$ with no variant, and $5 \%$ of those with $B M P R 2$ variants [11]. What differed from the previous study was that centrilobular ground glass opacification was less frequent; however, interlobular septal thickening abnormalities were more commonly present among PAH patients with $B M P R 2$ variants in our observation. In other words, radiologic features at the time of diagnosis could not accurately determine the exact diagnosis and underlying genotype.

According to 2015 ERS/ESC guidelines, low CI and $\mathrm{SvO}_{2}$ are associated with poor outcomes [1]. However, in the present study, we found no association between hemodynamic variables and primary outcomes. This might be ascribed to the following possible reasons. First, the sample size of the study was relatively small, but continuing patients are still enrolling. Besides, we used variables at the time of diagnosis when patients might have normal $\mathrm{CI}$ and/or $\mathrm{SvO} 2$ and we don't have any follow up information on hemodynamic. However, we noted lower Peak VO2\% and shorten 6MWD were associated with poor survival in our study. All these observations might imply exercise capacity decrease before hemodynamic deteriorating.

\section{Limitation}

There are some limitations to our study. On the one hand, the sample size was relatively small, which might inevitably weaken the force of our conclusions. However, PAHs are orphan diseases, and we are continuing to enroll new $\mathrm{PAH}$ patients to verify the present findings. In addition, we compared PAH patients with BMPR2 or biallelic EIF2AK4 variants without pathological examination. Current guidelines indicate that a diagnosis of heritable $\mathrm{PVOD} / \mathrm{PCH}$ can be made by identifying pathogenic biallelic EIF2AK4 variants without necessitating lung biopsy $[9,18]$, which may pose a substantial risk for morbidity or mortality for a patient with severe pulmonary hypertension and/or right ventricular failure. Therefore, we used strict clinical criteria, including high-resolution CT and DLCO, to analyse the phenotypes of these PAHs.

\section{Conclusion}

Our study reports the clinical and radiological features together with survival in a Chinese cohort diagnosed with $\mathrm{PAH}$, with the genetic background of BMPR2 and biallelic EIF2AK4 variants. The overall clinical characteristics of PAH patients with $B M P R 2$ and biallelic EIF2AK4 variants in Chinese patients were similar to those of other populations. However, a few characteristics were noteworthy:
Chinese PAH patients presented with a younger age at diagnosis compared with other populations. In addition, a female predominance among PAH patients with biallelic EIF2AK4 variants was also noted. All of these discrepancies may be due to geographic and race-specific factors, and some environmental elements may also play a role. However, further investigations are still warranted. Highresolution $\mathrm{CT}$ reveals a high incidence of radiological abnormalities in $\mathrm{PAH}$ patients with biallelic EIF2AK4 variants. However, similar radiological abnormalities can also be found in some $B M P R 2$ variant carriers. $B M P R 2$ variants and biallelic EIF2AK4 variants are associated with adverse outcomes, but the survival of patients with biallelic EIF2AK4 variants is dismal. As a result, genetic testing with high-resolution CT may assist in the identification of $\mathrm{PVOD} / \mathrm{PCH}$ in a timely manner and in referring for lung transplantation before their condition deteriorates.

\section{Supplementary information}

Supplementary information accompanies this paper at https://doi.org/10 1186/s12890-020-01179-7.

Additional file 1: Table S1 Pathogenic/Likely Pathogenic BMPR2 variants. Table S2 Pathogenic/Likely Pathogenic biallelic EIF2AK4 mutations

\section{Abbreviations}

PAH: Pulmonary arterial hypertension; BMPR2: Bone morphogenetic protein type 2 receptor; IPAH: Idiopathic pulmonary arterial hypertension;

HPAH: Heritable pulmonary arterial hypertension; PVOD/ PCH: Pulmonary venoocclusive disease/ pulmonary capillary haemangiomatosis; EIF2AK4: Eukaryotic

translation initiation factor 2 alpha kinase 4; KCNK3: Potassium two-pore domain channel subfamily K member 3; CAV1: Caveolin 1; SMAD9: SMAD family member 9; BMPR1B: Bone morphogenetic protein receptor type 1B; ACVRL1: Activin A receptor-like type 1; ENG: Endoglin; GDF2: Growth differentiation factor 2; SMAD4: SMAD family member 4; KCNA5: Potassium voltage-gated channel subfamily A member 5; NOTCH3: Notch 3; TOPBP1: DNA Topoisomerase II binding protein 1; mPAP: Mean pulmonary arterial pressure; PCWP: Pulmonary capillary wedge pressure; PVR: Pulmonary vascular resistance; SvO2: Mixed venous oxygen saturation; SaO2: Arterial oxygen saturation; CO: Cardiac output; NT-proBNP: N-terminal pro-B-type natriuretic peptide; 6MWD: 6-min walk distance; DLOC: Diffusing lung capacity of carbon monoxide; Cl: Cardiac index; CT: computed tomography

\section{Acknowledgements}

Not applicable.

\section{Authors' contributions}

CMX is the guarantor of the article. CMX and ZZ contributed to conceiving and designing this study. XQZ contributed to recruiting patients, analyzing the clinical data, drafting and revising the manuscript. HY contributed to interpreting the data and revising the manuscript. BYL contributed to analyzing data and drafting the manuscript. ZHL contributed to recruiting patients and collecting clinical data. YYM and WKL contributed to performing sequencing and MLPA. QLC, ZHZ and QL were in charge of sample handling and quality control. All authors contributed to reviewing and approved the final manuscript.

\section{Funding}

The present study was financially supported by the grant of Capital Health Development and Scientific Research Projects (2016-2-4036) and CAMS Initiative for Innovative Medical (2016-I2M-3-006). 


\section{Availability of data and materials}

The datasets used and/or analyzed during the current study are available from the corresponding author on reasonable request.

\section{Ethics approval and consent to participate}

Each individual accepting the genetic test was informed and signed a consent form. The study was approval by the ethics committee of Fuwai Hospital (Approval NO: 2017-877) and adhered to the Declaration of Helsinki.

\section{Consent for publication}

All individual accepting the genetic test signed a consent form and agreed to allow their anonymized samples and results used for further research and publications.

\section{Competing interests}

All the authors have no conflicts of interest to declare.

Received: 27 December 2019 Accepted: 11 May 2020

Published online: 29 May 2020

\section{References}

1. Galie N, Humbert M, Vachiery JL, et al. 2015 ESC/ERS Guidelines for the diagnosis and treatment of pulmonary hypertension: The Joint Task Force for the Diagnosis and Treatment of Pulmonary Hypertension of the European Society of Cardiology (ESC) and the European Respiratory Society (ERS): Endorsed by: Association for European Paediatric and Congenital Cardiology (AEPC), International Society for Heart and Lung Transplantation (ISHLT). Eur Heart J. 2016;37:67-119. https://doi.org/10.1093/eurheartj/ ehv317.

2. Liu D, Liu QQ, Eyries $M$, Wu WH, Yuan P, Zhang R, Soubrier F, Jing ZC. Molecular genetics and clinical features of Chinese idiopathic and heritable pulmonary arterial hypertension patients. Eur Respir J. 2012;39:597-603. https://doi.org/10.1183/09031936.00072911.

3. Evans JDW, Girerd B, Montani D, et al. BMPR2 mutations and survival in pulmonary arterial hypertension: an individual participant data metaanalysis. Lancet Respir Med. 2016;4:129-37. https://doi.org/10.1016/s22132600(15)00544-5.

4. $\quad$ Yang H, Zeng Q, Ma Y, Liu B, Chen Q, Li W, Xiong C, Zhou Z. Genetic analyses in a cohort of 191 pulmonary arterial hypertension patients. Respir Res. 2018;19:87. https://doi.org/10.1186/s12931-018-0789-9.

5. Morrell NW, Aldred MA, Chung WK, Elliott CG, Nichols WC, Soubrier F, Trembath RC, Loyd JE. Genetics and genomics of pulmonary arterial hypertension. Eur Respir J. 2019;53:1. https://doi.org/10.1183/13993003. 01899-2018.

6. Runo JR, Loyd JE. Primary pulmonary hypertension. Lancet. 2003;361:153344. https://doi.org/10.1016/s0140-6736(03)13167-4

7. Montani $D$, Achouh $L$, Dorfmuller $P$, et al. Pulmonary veno-occlusive disease: clinical, functional, radiologic, and hemodynamic characteristics and outcome of 24 cases confirmed by histology. Medicine (Baltimore). 2008;87: 220-33. https://doi.org/10.1097/MD.0b013e31818193bb.

8. Best DH, Sumner $\mathrm{KL}$, Austin ED, et al. EIF2AK4 mutations in pulmonary capillary hemangiomatosis. Chest. 2014;145:231-6. https://doi.org/10.1378/ chest.13-2366.

9. Eyries M, Montani D, Girerd B, et al. EIF2AK4 mutations cause pulmonary veno-occlusive disease, a recessive form of pulmonary hypertension. Nat Genet. 2014;46:65-9. https://doi.org/10.1038/ng.2844.

10. Best $\mathrm{DH}$, Sumner $\mathrm{KL}$, Smith BP, et al. EIF2AK4 mutations in patients diagnosed with pulmonary arterial hypertension. Chest. 2017;151:821-8. https://doi.org/10.1016/j.chest.2016.11.014

11. Hadinnapola C, Bleda M, Haimel M, et al. Phenotypic characterization of EIF2AK4 mutation carriers in a large cohort of patients diagnosed clinically with pulmonary arterial hypertension. Circulation. 2017;136:2022-33. https:// doi.org/10.1161/circulationaha.117.028351.

12. Montani D, Girerd B, Jais X, et al. Clinical phenotypes and outcomes of heritable and sporadic pulmonary veno-occlusive disease: a populationbased study. Lancet Respir Med. 2017;5:125-34. https://doi.org/10.1016/ s2213-2600(16)30438-6.

13. McGoon MD, Krichman A, Farber HW, Barst RJ, Raskob GE, Liou TG, Miller DP, Feldkircher K, Giles S. Design of the REVEAL registry for US patients with pulmonary arterial hypertension. Mayo Clin Proc. 2008;83:923-31. https:// doi.org/10.4065/83.8.923.

14. Frost $A E$, Badesch DB, Barst RJ, et al. The changing picture of patients with pulmonary arterial hypertension in the united state: how REVEAL differs from historic and non-US contemporary registries. Chest. 2011;139:128-37. https://doi.org/10.1378/chest.10-0075.

15. Austin ED, Loyd JE. Heritable forms of pulmonary arterial hypertension. Semin Respir Crit Care Med. 2013;34:568-80. https://doi.org/10.1055/s-00331355443.

16. Pousada G, Baloira A, Fontan D, Nunez M, Valverde D. Mutational and clinical analysis of the ENG gene in patients with pulmonary arterial hypertension. BMC Genet. 2016;17:72. https://doi.org/10.1186/s12863-0160384-3.

17. Ali N, Loughborough WW, Rodrigues JCL, Suntharalingam J, Hudson B, Hall T, Augustine D, Mackenzie R, Robinson G. Computed tomographic and clinical features of pulmonary veno-occlusive disease: raising the radiologist's awareness. Clin Radiol. 2019;S0009-9260(19):30221. https://doi org/10.1016/j.crad.2019.04.023.

18. Condon DF, Nickel NP, Anderson R, Mirza S, de Jesus PVA. The $6^{\text {th }}$ world symposium on pulmonary hypertension: what's old is new. F1000Res 8; 2019. https://doi.org/10.12688/f1000research.18811.1. eCollection 2019.

\section{Publisher's Note}

Springer Nature remains neutral with regard to jurisdictional claims in published maps and institutional affiliations.
Ready to submit your research? Choose BMC and benefit from:

- fast, convenient online submission

- thorough peer review by experienced researchers in your field

- rapid publication on acceptance

- support for research data, including large and complex data types

- gold Open Access which fosters wider collaboration and increased citations

- maximum visibility for your research: over $100 \mathrm{M}$ website views per year

At BMC, research is always in progress.

Learn more biomedcentral.com/submissions 